\title{
CORRECTION
}

\section{Correction: Clinical review: Prothrombin complex concentrates - evaluation of safety and thrombogenicity}

\author{
Benny Sørensen ${ }^{1 *}$, Donat R Spahn², Petra Innerhofer ${ }^{3}$, Michael Spannag ${ }^{4}$ and Rolf Rossaint ${ }^{5}$ \\ See related review by Sørensen et al., http://ccforum.com/content/15/1/201
}

\section{Correction}

After publication of our article [1] we noted that in Table 1 for Prothrombinex-VF 'Antithrombin and heparin added' should have been included under the 'Additional information' column. A copy of the table with the correct information can be found overleaf.

\section{Author details}

'Haemostasis Research Unit, Centre for Haemostasis and Thrombosis, Department of Haematology and Oncology, Guy's and St Thomas' Hospital \& NHS Trust Foundation, King's College London School of Medicine, 1st Floor, North Wing, St Thomas' Hospital, Westminster Bridge Road, London SE1 7EH, UK. Institute of Anesthesiology, University Hospital Zurich, CH-8091 Zurich, Switzerland. ${ }^{3}$ Department of Anaesthesiology and Critical Care Medicine, Innsbruck Medical University, Anichstrasse 35, A-6020 Innsbruck, Austria. ${ }^{4}$ Working Group on Perioperative Hemostasis, Department of Anaesthesiology, University Hospital of Munich, Nussbaumstrasse 20, 80336 Munich, Germany. ${ }^{5}$ Department of Anaesthesiology, University Hospital Aachen, RWTH Aachen University, Pauwelsstrasse 30, 52074 Aachen, Germany.

\section{Published: 18 March 2011}

\section{Reference}

1. Sørensen B, Spahn DR, Innerhofer P, SpannagI M, Rossaint R: Clinical review: Prothrombin complex concentrates - evaluation of safety and thrombogenicity. Crit Care 2011, 15:201.

doi:10.1186/cc10094

Cite this article as: Sørensen B, et al.: Correction: Clinical review: Prothrombin complex concentrates - evaluation of safety and thrombogenicity. Critical Care 2011, 15:409.
*Correspondence: benny.sorensen@kcl.ac.uk

'Haemostasis Research Unit, Centre for Haemostasis and Thrombosis, Department of Haematology and Oncology, Guy's and St Thomas' Hospital \& NHS Trust

Foundation, King's College London School of Medicine, 1st Floor, North Wing,

St Thomas' Hospital, Westminster Bridge Road, London SE1 7EH, UK

Full list of author information is available at the end of the article 
Table 1. Composition of PCCs in the World Federation of Hemophilia register of clotting factor concentrates

\begin{tabular}{|c|c|c|c|c|c|c|c|}
\hline \multirow[b]{2}{*}{ Brand name } & \multirow[b]{2}{*}{ Manufacturer } & \multicolumn{4}{|c|}{ International units relative to factor IX } & \multirow[b]{2}{*}{ Viral inactivation } & \multirow[b]{2}{*}{$\begin{array}{l}\text { Additional } \\
\text { information }\end{array}$} \\
\hline & & $\begin{array}{c}\text { Factor } \\
\text { II }\end{array}$ & $\begin{array}{l}\text { Factor } \\
\text { VII }\end{array}$ & $\begin{array}{l}\text { Factor } \\
\text { IX }\end{array}$ & $\begin{array}{l}\text { Factor } \\
\mathrm{X}\end{array}$ & & \\
\hline Bebulin VH & $\begin{array}{l}\text { Baxter BioScience, } \\
\text { Austria }\end{array}$ & 120 & (13) & 100 & 100 & $\begin{array}{l}\text { Vapour heat, } 60^{\circ} \mathrm{C} \text { for } 10 \text { hours at } 190 \mathrm{mbar} \text {, } \\
\text { then } 80^{\circ} \mathrm{C} \text { for } 1 \text { hour at } 375 \mathrm{mbar}\end{array}$ & Heparin added \\
\hline Beriplex P/N & $\begin{array}{l}\text { CSL Behring, } \\
\text { Germany }\end{array}$ & 128 & 68 & 100 & 152 & $\begin{array}{l}\text { Pasteurisation at } 60^{\circ} \mathrm{C} \text { for } 10 \text { hours, and } \\
\text { nanofiltration }\end{array}$ & $\begin{array}{l}\text { Protein } C_{i} \\
\text { antithrombin, heparin } \\
\text { and albumin added }\end{array}$ \\
\hline Cofact & $\begin{array}{l}\text { Sanquin, } \\
\text { the Netherlands }\end{array}$ & $56-140$ & $28-80$ & 100 & $56-140$ & $\begin{array}{l}\text { Solvent/detergent and } 15 \mathrm{~nm} \\
\text { nanofiltration }\end{array}$ & Antithrombin added \\
\hline KASKADIL & LFB, France & 148 & 40 & 100 & 160 & Solvent/detergent & Heparin added \\
\hline Octaplex & $\begin{array}{l}\text { Octapharma, Austria } \\
\text { and France }\end{array}$ & $44-152$ & $36-96$ & 100 & 50 & Solvent/detergent and nanofiltration & $\begin{array}{l}\text { Heparin added; low } \\
\text { activated factor VII } \\
\text { content }\end{array}$ \\
\hline Profilnine SD & Grifols, USA & 148 & (11) & 100 & 64 & Solvent/detergent & - \\
\hline Prothrombinex VF & $\begin{array}{l}\text { CSL Bioplasma, } \\
\text { Australia }\end{array}$ & 100 & $(-)$ & 100 & 100 & $\begin{array}{l}\text { Dry heat, } 80^{\circ} \mathrm{C} \text { for } 72 \text { hours and } \\
\text { nanofiltration }\end{array}$ & $\begin{array}{l}\text { Antithrombin and } \\
\text { heparin added }\end{array}$ \\
\hline Prothromplex $T$ & $\begin{array}{l}\text { Baxter BioScience, } \\
\text { Austria }\end{array}$ & 100 & 85 & 100 & 100 & $\begin{array}{l}\text { Vapour heat, } 60^{\circ} \mathrm{C} \text { for } 10 \text { hours at } 190 \mathrm{mbar} \text {, } \\
\text { then } 80^{\circ} \mathrm{C} \text { for } 1 \text { hour at } 375 \mathrm{mbar}\end{array}$ & $\begin{array}{l}\text { Antithrombin and } \\
\text { heparin added }\end{array}$ \\
\hline UMAN Complex D.I. & Kedrion, Italy & 100 & $(-)$ & 100 & 80 & $\begin{array}{l}\text { Solvent/detergent and dry heat, } 100^{\circ} \mathrm{C} \text { for } \\
30 \text { minutes }\end{array}$ & $\begin{array}{l}\text { Antithrombin and } \\
\text { heparin added }\end{array}$ \\
\hline
\end{tabular}

Composition of prothrombin complex concentrates (PCCs) listed in the World Federation of Hemophilia register of clotting factor concentrates [54], excluding concentrates for national markets only or for which the concentrations of factors relative to factor IX were not available from the relevant product information sheets. Factor VII presented in parentheses for three-factor PCCs. 